\title{
EFEITO DA IDADE RELATIVA NO FUTSAL DE BASE DE MINAS GERAIS
}

\author{
GRAD. EDUARDO MACEDO PENNA \\ Mestrando do Programa de Pós-Graduação em Ciências do Esporte - Escola de Educação Física, \\ Fisioterapia e Terapia Ocupacional/Universidade Federal de Minas Gerais \\ Laboratório de Psicologia do Esporte - LAPES/UFMG \\ (Belo Horizonte - Minas Gerais - Brasil) \\ Email: em.penna@yahoo.com.br
}

MS. VARLEY TEOLDO DA COSTA

Mestre em Ciências do Esporte - Escola de Educação Física, Fisioterapia e

Terapia Ocupacional/Universidade Federal de Minas Gerais

Professor do Centro Universitário de Belo Horizonte - UNIBH

Laboratório de Psicologia do Esporte - LAPES/UFMG

(Belo Horizonte - Minas Gerais - Brasil)

Email: vtcosta@hotmail.com

MS. RENATO MELO FERREIRA

Mestre em Ciências do Esporte -Escola de Educação Física, Fisioterapia e

Terapia Ocupacional/Universidade Federal de Minas Gerais

Professor do Centro Universitário de Belo Horizonte - UNIBH

Laboratório de Psicologia do Esporte - LAPES/UFMG

(Belo Horizonte - Minas Gerais - Brasil)

Email: renato.mf@hotmail.com

\section{DR. LUIZ CARLOS COUTO DE ALBUQUERQUE MORAES}

Doutor em Psicopedagogia University of Ottawa, Canadá. Professor Associado II - Escola de

Educação Física, Fisioterapia e Terapia Ocupacional/Universidade Federal de Minas Gerais

Laboratório de Psicologia do Esporte - LAPES/UFMG

(Belo Horizonte - Minas Gerais - Brasil)

Email:Imoraes@eeffto.ufmg.br 


\section{RESUMO}

O Efeito da Idade Relativa, representado pela vantagem obtida pelo atleta nascido mais próximo ao início do ano de seleção, mostra-se uma variável significativa para a identificação de talentos em diversas modalidades. Objetivou-se avaliar o quartil de nascimento de atletas das categorias de base do futsal em Minas Gerais. Foram analisados 510 atletas inscritos em competições federadas em 2009. Para análise empregou-se o teste de qui-quadrado. Verificou-se diferenças significativas na distribuição dos quartis de nascimento em quatro das cinco categorias avaliadas, com predominâncias do primeiro e segundo quartis. Concluiu-se que o Efeito da Idade Relativa é um fator que pode influenciar a seleção de atletas nas categorias de base do futsal.

PALAVRAS-CHAVE: Talento; futsal; grupos etários; efeito da idade relativa.

\section{INTRODUÇÃO}

○ futsal é uma modalidade esportiva de grande aceitação no Brasil e também no mundo. Sabe-se que mais de dez milhões de pessoas praticam o futsal ao redor do planeta (DACOSTA, 2005). Essa modalidade também contribui de forma incisiva na formação de futuros atletas para o futebol de campo (MARQUES; SAMULSKI, 2009). Contudo, é dentro do contexto escolar que o futsal se faz marcante, sendo a principal modalidade praticada por meninas e meninos dentro da escola (GENEROSI et al., 2008; QUEIROGA; FERREIRA; ROMANZINI, 2005).

Diante das demandas fisiológicas exigidas em uma partida de futsal, seja ela de alto nível ou em suas categorias de base, os esforços determinantes para o resultado são oriundos da velocidade, agilidade e potência muscular (ARAÚJO et al., 1996). Logo, o jogo é caracterizado por intensos esforços em um curto intervalo de tempo. Consequentemente, além das características técnicas e táticas, as características físicas e antropométricas dos jogadores de futsal são fundamentais para o sucesso das equipes (QUEIROGA; FERREIRA; ROMANZINI, 2005). Neste cenário, fica claro que os atletas de maior vigor físico terão vantagens sobre aqueles de menor vigor na disputa pelo espaço nas melhores equipes de futsal, especialmente em idades mais jovens.

Glamser e Vicent (2004) denominaram como "Efeito da Idade Relativa" essa possível vantagem que os atletas nascidos mais próximos ao início do ano de seleção levam em relação a seus pares nascidos posteriormente. A presença do Efeito da Idade Relativa já foi comprovada em diversas modalidades, como tênis (EDGAR; O'DONOGHUE, 2006), basquetebol (DELORME; RASPAUD, 2009), beisebol (TOMPSON; BARNSLEY; STEBELSKY, I99I) hóquei no gelo (SHERAR et al., 2007), futebol americano (CÔTÉ et al., 2006; MACDONALD et al., 2009) e principalmente futebol (MORAES et al., 2009; COSTA et al., 2009, JIMÉNEZ; PAIN, 
2008, FOLGADO et al., 2006). Para a análise dessa variável, os pesquisadores têm empregado a categorização do quartil de nascimento, que é considerada a divisão do ano em quatro partes. No caso do futsal, o primeiro quartil representa os meses de Janeiro a Março; o segundo, de Abril a Junho; o terceiro, de Julho a Setembro; e o quarto e último quartil, de Outubro a Dezembro (VAEYENS; PHILIPPAERTS; MALINA, 2005; MUSCH; HAY, 1999).

A influência do Efeito da Idade Relativa na seleção de atletas para as categorias de base, pode ser demonstrada conforme estudo de Helsen, Starkes e Winckel (2000). Os autores verificaram que a mudança na linha divisória imposta pela federação belga de futebol, para as categorias de base, passando do dia primeiro de agosto para o dia primeiro de janeiro, alterou significativamente a representação dos jogadores nascidos em cada quartil. Anteriormente a essa mudança, os jogadores nascidos em agosto, setembro e outubro (primeiro quartil) e novembro, dezembro e janeiro (segundo quartil), representavam a maioria dos atletas da categoria 10 a 12 anos. Dois anos após a mudança, observou-se que a proporção de atletas nascidos em janeiro, fevereiro e março (novo primeiro quartil) e abril, maio e junho (novo segundo quartil) passou a ser mais representativo estatisticamente.

Outro fator de grande importância relacionado com o Efeito da Idade Relativa em jovens atletas, diz respeito ao abandono da modalidade. Delorme, Boiché e Raspaud (2010), descrevem que, em cinco das sete categorias de base do futebol francês (sub 9, sub I I, sub |3, sub I 5 e sub |8), os jogadores nascidos no segundo semestre do ano de seleção abandonam (dropout) mais a modalidade, quando comparados com aqueles nascidos nos dois primeiros quartis do ano. Logo, os autores concluem que o Efeito da Idade Relativa pode atuar como um fator de abandono da modalidade, haja visto que as condições técnicas, táticas, psicológicas e, principalmente físicas, são desiguais entre os atletas de uma mesma faixa etária, em função dos desequilíbrios proporcionados pela idade relativa.

Contudo, não é somente a diferença física entre os atletas de uma mesma categoria que pode influenciar o abandono por parte do jovem desfavorecido pelo Efeito da Idade Relativa. Os fatores psicológicos, como os níveis de autoconfiança e das percepções sobre o próprio desempenho, que nem sempre são citados nesse tipo de estudo, podem também prejudicar o processo de seleção de atletas (SIMMONS; PAULL, 200 I). Musch e Grondin (200 I) citam o impacto negativo na auto-estima e nos níveis motivacionais que podem ser gerados nos atletas preteridos, quando existe o favorecimento por aqueles nascidos anteriormente.

Portanto, dada a importância da modalidade para a formação de atletas em idade escolar, e devido à escassez de estudos envolvendo essa modalidade, esse trabalho teve como objetivo: I) analisar a distribuição de atletas de futsal das categorias de base 
e adulta de futsal do estado de Minas Gerais e 2) verificar possíveis assimetrias nessa distribuição e a presença do Efeito da Idade Relativa nessa população.

\section{MATERIAIS E MÉTODOS}

\section{AMOSTRA}

A amostra foi composta por 510 atletas, todos do gênero masculino, pertencentes a todas as 23 equipes filiadas da Federação Mineira de Futsal que disputaram algum torneio oficial regulamentado pela referida federação, no ano de 2009 (campeonato metropolitano ou campeonato do interior do estado). Todas as categorias competitivas foram analisadas: sub || $(n=90)$, sub $\mid 3(n=\mid 63)$, sub I $5(n=103)$, sub $17(n=129)$, sub 20/Adulto $(n=125)$, sendo que as duas últimas foram analisadas em conjunto, pois as equipes sub 20 e adultas disputam o mesmo campeonato, sem distinção etária.

Este estudo foi aprovado pelo Comitê de Ética da Universidade Federal de Minas Gerais, sob o protocolo número ETIC I 28/08.

\section{PROCEDIMENTOS}

Primeiramente foi realizado um contato com a Federação Mineira de Futsal (FMFS), para a explicitação do método e objetivos da pesquisa. Após a aprovação por parte da direção executiva da entidade, foram coletadas, dentro do banco de dados da FMFS, as informações referentes às datas de nascimento dos atletas e o clube ao qual os mesmos estavam vinculados. Esses dados foram tabulados em uma planilha própria para a posterior análise estatística.

\section{ANÁLISE ESTATIÍSTICA}

Com relação à análise do Efeito da Idade Relativa, foi realizado o teste de Qui-Quadrado para a comparação das diferenças entre os quartis. No caso do Futsal, adota-se como primeiro quartil os meses de Janeiro, Fevereiro e Março; o segundo quartil representado pelos meses de Abril, Maio e Junho; o terceiro quartil representado pelos meses de Julho, Agosto e Setembro; e o quarto quartil representado pelos meses de Outubro, Novembro e Dezembro. Foi adotado um nível de significância de 5\%. Uma posterior análise de proporção 2×2 com correção de Bonferroni foi aplicada para averiguar onde estavam as diferenças encontradas. A correção de Bonferroni, que altera o nível de significância ( $p)$, foi utilizada a fim de evitar erros derivados de múltiplas comparações. $\bigcirc$ nível de significância corrigido para a análise de proporção $2 \times 2$ foi de $p<0.0083$. 
Todos os procedimentos foram feitos utilizando-se o programa SPSS 17.0 para Windows.

\section{RESULTADOS}

De acordo com a faixa etária de cada atleta, o Gráfico I apresenta, dentro das 5 categorias federadas do futsal mineiro, a distribuição dos jogadores pelo quartil de nascimento.

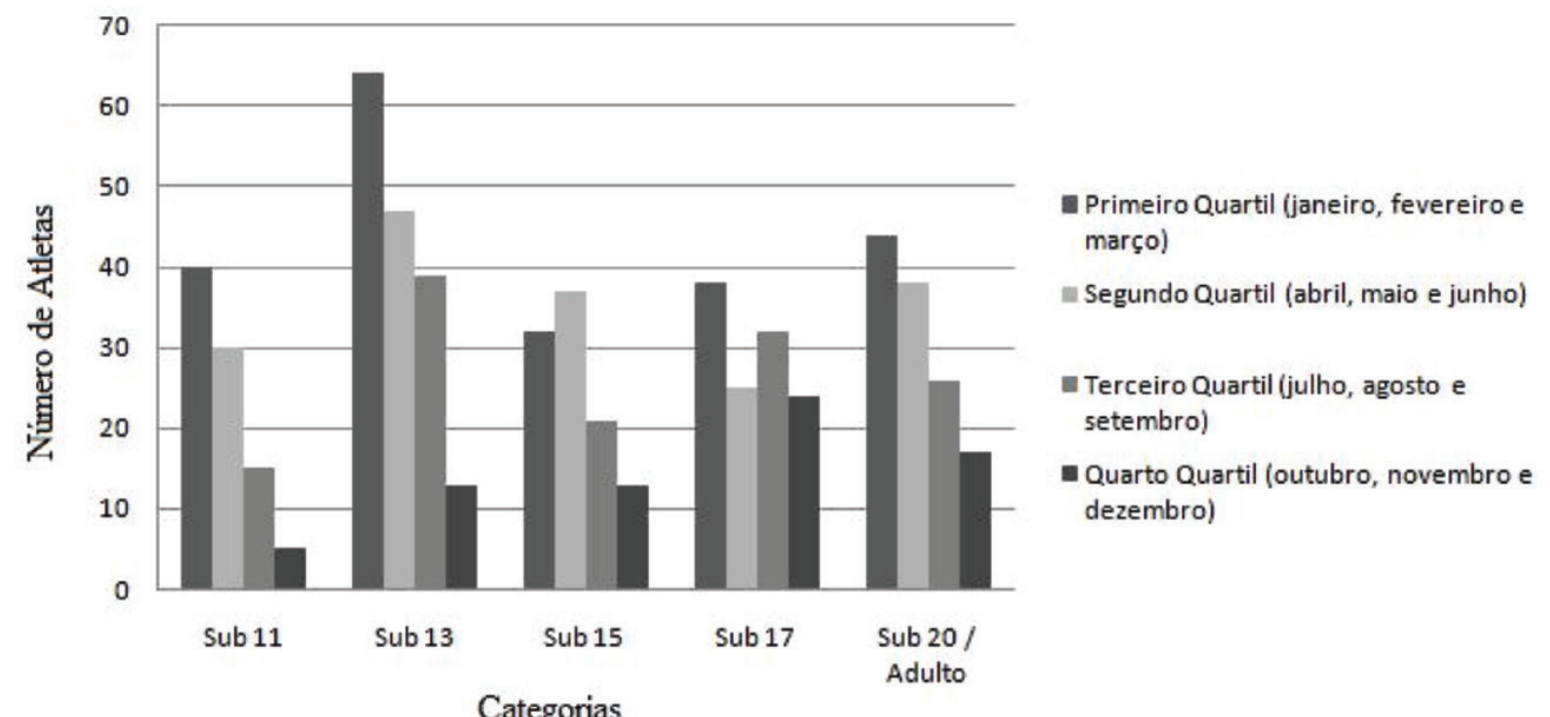

Gráfico I. Distribuição dos quartis de nascimento dos atletas de futsal ativos no ano de 2009 no estado de Minas Gerais.

Conforme demonstrado no Gráfico I no Gráfico I, podemos observar que o quarto quartil é o menos representativo em todas as categorias. Outro ponto interessante é que apenas na categoria sub- 15 o primeiro quartil não apresenta a maior representatividade absoluta.

Os dados apresentados na Tabela I referem-se aos resultados do teste de Qui-quadrado para cada categoria etária. Apenas para a categoria sub 17 não foram encontradas diferenças significativas.

Tabela I. Comparação do quartil de nascimento nas diferentes categorias do futsal

\begin{tabular}{llllll}
\hline & Sub II & Sub 13 & Sub I5 & Sub 17 & Sub 20 / Adulto \\
\hline Primeiro Quartil & 40 & 64 & 32 & 38 & 44 \\
Segundo Quartil & 30 & 47 & 37 & 25 & 38 \\
Terceiro Quartil & 15 & 39 & 21 & 32 & 26 \\
Quarto Quartil & 5 & 13 & 13 & 24 & 17 \\
$X^{2}$ & 32,222 & 33,196 & 13,621 & 4,328 & 14,040 \\
$p$ & $p=0,000$ I* & $p=0,000$ I* & $p=0,003 *$ & $p=0,228$ & $P=0,003 *$ \\
\hline
\end{tabular}

$*_{p}<0,05$ 
Após a realização de análise de proporção 2×2 com Correção de Bonferroni, entre os quartis de nascimento de cada categoria (onde foram encontradas diferenças no teste de Qui-Quadrado), em todas elas foram encontradas diferenças entre o primeiro e quarto quartil, e entre o segundo e o quarto quartil, demonstrando, assim, uma baixa representatividade do quarto quartil em relação ao primeiro e segundo. Quando divididos por semestres $\left(1^{\circ}\right.$ e $2^{\circ}$ quartis versus $3^{\circ}$ e $4^{\circ}$ quartis), exceto para a categoria sub 17, as diferenças encontradas apontam para uma maior representatividade do primeiro semestre e, consequentemente, uma sub representatividade do segundo, principalmente sobre os atletas nascidos no último quartil, que foram significativamente menos representados do que os atletas do primeiro e do segundo quartis, conforme representado pelas Tabelas 2 e 3.

Tabela 2. Comparação entre os quartis de nascimento nas diferentes categorias do futsal através da análise de proporção

\begin{tabular}{lllllll}
\hline & $1^{\circ} \times 2^{\circ}$ & $1^{\circ} \times 3^{\circ}$ & $1^{\circ} \times 4^{\circ}$ & $2^{\circ} \times 3^{\circ}$ & $2^{\circ} \times 4^{\circ}$ & $3^{\circ} \times 4^{\circ}$ \\
\hline Sub II & 0,124 & $0,000 I^{*}$ & $0,000 I^{*}$ & 0,009 & $0,000 I^{*}$ & 0,015 \\
Sub I3 & 0,046 & $0,003^{*}$ & $0,000 I^{*}$ & $0,3 \mid 4$ & $0,000 I^{*}$ & $0,000 I^{*}$ \\
Sub 15 & 0,136 & 0,077 & $0,00 I^{*}$ & 0,012 & $0,000 I^{*}$ & 0,131 \\
Sub 20 /Adulto & $0,4 \mid 8$ & 0,010 & $0,000 I^{*}$ & 0,080 & $0,00 I^{*}$ & 0,130 \\
\hline
\end{tabular}
$*_{p}<0,0083$

Tabela 3. Comparação entre os semestres de nascimento nas diferentes categorias do futsal

\begin{tabular}{|c|c|c|c|c|c|}
\hline & Sub II & Sub 13 & Sub 15 & Sub 17 & Sub 20 / Adulto \\
\hline Primeiro Semestre & 70 & || $\mid$ & 69 & 63 & 82 \\
\hline Segundo Semestre & 20 & 52 & 34 & 66 & 43 \\
\hline $\begin{array}{l}X^{2} \\
p\end{array}$ & $\begin{array}{l}27,778 \\
p=0,000 \mid *\end{array}$ & $\begin{array}{l}21,356 \\
p=0,000 \mid *\end{array}$ & 11,893 & $\begin{array}{l}0,700 \\
p=0,792\end{array}$ & $\begin{array}{l}12,168 \\
P=0,000 \mid *\end{array}$ \\
\hline
\end{tabular}

$*_{p}<0,05$

\section{DISCUSSÃO}

Os resultados encontrados pelo presente estudo comprovam a existência do Efeito da Idade Relativa nas categorias de base e adulta no futsal do estado de Minas Gerais, e a consequente influência desta variável na seleção de atletas das equipes federadas. Entretanto, para efeitos de discussão, não será possível comparar os resultados deste trabalho com outros estudos na modalidade futsal, devido à escassez de publicações nesta área. Assim, adotou-se como critério a realização desta discussão confrontando os achados do futsal com os achados da modalidade futebol de campo. Haja visto que, assim como outras modalidades, ambas são 
classificadas como jogos de confronto, que desenvolvem suas ações em um espaço comum e com participação sobre a bola, de forma simultânea (MORALES et al., 2009), e que possuem uma essência de jogo muito semelhante no que tange aos princípios técnicos táticos, físicos e psicológicos. Além disso, segundo Marques e Samulski (2009), normalmente, o jogador de futsal passa por este estágio seletivo antes de se tornar um jogador de futebol de campo.

Conforme citado anteriormente, diversos fatores influenciarão o progresso de um jovem ao alto nível de desempenho no futsal. Contudo, o impacto de variáveis psicológicas e cognitivas é tão importante quanto as físicas e técnico-táticas. Musch e Grondin (200I ) citam que, quanto mais alta for a percepção de competência de uma criança, maior será a motivação intrínseca e o prazer pela prática por parte dela. Logo, ainda segundo esses autores, aquelas crianças favorecidas pelo Efeito da Idade Relativa possuem maiores chances de continuar praticando a modalidade e, assim, continuar desenvolvendo-se técnica e taticamente. Fica claro, nesse ponto, que o Efeito da Idade Relativa no futsal, cuja estruturação é feita em 24 meses (primeiro e segundo ano de categoria), é um fator que pode influenciar a permanência das crianças na modalidade que, segundo demonstrado pelos resultados do presente estudo, também apresenta esse efeito em suas categorias de base e adulta.

Corroborando os resultados supracitados, Helsen, Winckel e Williams (2005) citam que, quanto melhor for o rendimento inicial percebido pela criança, maior a motivação intrínseca dela. Logo, como as crianças nascidas no início do ano de seleção tendem a ter um desempenho inicial melhor, maior será a motivação dessas crianças para permanecerem na modalidade e continuarem a se desenvolver, técnica e taticamente. Isso pode, em parte, explicar a discrepante distribuição das datas de nascimento das categorias menores, principalmente sub | | e sub |3, onde as diferenças físicas e cognitivas tendem a ser maiores, devido principalmente ao início do processo pubertário.

Este tipo de seleção natural, no qual as crianças que amadureceram mais rapidamente durante a puberdade, levam vantagens sobre as crianças que demoram um pouco mais a manifestar seus caracteres secundários de desenvolvimento, acaba por criar um sistema desigual no processo de seleção de novos talentos, pois a exclusão ou a inclusão de um atleta dentro da equipe de futsal passa por uma avaliação do seu rendimento biológico, físico, técnico, tático, psicológico e social. Essa relação desigual pode ser demonstrada conforme o estudo de Vitor et al. (2008), que concluiu que a maturação biológica teve efeito significativo sobre a potência de membros inferiores, superiores e na velocidade de deslocamento em crianças de 12 a 16 anos. Efeito esse que pode ser determinante na inclusão ou exclusão de um jovem em uma equipe. 
Com isso, crianças do terceiro e quarto quartis têm estatisticamente suas chances reduzidas de atingirem o alto rendimento no futsal. Nas fases iniciais de seleção por parte dos clubes (categorias sub- I I, sub- I 3 e sub- I 5), o efeito causado pela variável da idade relativa pode provocar o abandono da modalidade (DELORME; BOICHÉ; RASPAUD, 20 I0). Pode, também, levar este jovem atleta a ter toda a sua formação em clubes de menor expressão (não federados), que não estão envolvidos em competições federadas, onde se busca a excelência na formação e no nível de rendimento e competitividade dos atletas (MORAES et al., 2009).

Outro ponto apresentado por Gil et al. (2007) diz respeito à estruturação das categorias, já que, ao se agrupar os jogadores em categorias que duram dois anos, cria-se uma enorme diferença física entre aqueles nascidos no início do primeiro ano competitivo e aqueles nascidos no final do segundo ano. Esse segundo grupo possui maiores chances de serem identificados como não talentosos, e tendem a abandonar a modalidade. Essa estrutura, que também se faz presente em todas as categorias de base do futsal, pode estar potencializando o Efeito da Idade Relativa, conforme demonstrado pelos resultados do presente estudo.

Apesar de algumas propostas para a atenuação das consequências deste efeito ser de difícil aplicabilidade na prática, dentro do ambiente competitivo do futsal o conhecimento desta variável, principalmente por membros da comissão técnica e por dirigentes de categorias de base, se faz necessário. Cabe, aos mesmos, a compreensão de que estas desvantagens físicas, técnicas, táticas e psicológicas podem ser temporárias, possibilitando, assim, uma futura disputa pelo espaço em equipes federadas mais adequadas.

\section{CONCLUSÃO}

Desta forma, conclui-se no presente estudo que a maioria dos atletas federados avaliados no estado de Minas Gerais nasceu no primeiro semestre do ano competitivo. Dentre as comparações realizadas entre os diferentes quartis, observam-se assimetrias nas diferentes categorias (com predominância de assimetria entre primeiro e quarto quartis.)

Este estudo avaliou somente a interferência da variável Idade Relativa no contexto de seleção de atletas de futsal, e teve como limitação a não mensuração do estado maturacional e do tempo de prática competitiva da modalidade. Contudo, vale ressaltar a importância da análise de outras variáveis (físicas, táticas e psicológicas) que interferem no processo de identificação, seleção e formação dessas equipes de futsal. 


\section{Relative age effect in futsal in Minas Gerais}

ABSTRACT:The effect of relative age, understood as the advantage obtained by athletes born in the beginning of the year of selection, is a significant variable for the development of talents in several modalities. Therefore, the objective is to assess the quartile of birth of athletes of the minor league categories of futsal in Minas Gerais. The sample was composed by 510 athletes enrolled in federated competitions in 2009. The chi-square test was used in this analysis. There were significant differences in the distribution of the quartiles of birth in four of the five categories assessed, with a predominance of the first and second quartiles. In conclusion, the effect of relative age is a factor which might influence the choice of futsal players in the base categories.

KEY-WORDS: Talent; futsal; age groups; relative age effect.

\section{El efecto de la edad relative en el fútbol sala de las categorias de base de Minas Gerais}

RESUMEN: El efecto de la edad relativa, entendido como la ventaja obtenida por el atleta nacido em las primeras fechas del año de selección, se muestra una variable significativa para el desarrollo de talentos en distintas modalidades. Por esta se evaluó el cuartil de nacimiento de atletas de las categorias base del fútbol sala em Minas Gerais. La muestra estaba compuesta por 510 atletas inscritos en competiciones federadas en 2009. Para este análisis fue empleado el test de chi-cuadrado. Fueron encontradas diferencias significativas en la distribucíon de los cuartiles de nacimiento em cuatro de las cinco categorias evaluadas, con predomínio del primer y segundo. Se concluyó que el efecto de la edad relativa es un factor que puede influenciar em la selección de jugadores de fútbol sala em las categorias de base. PALABRAS CLAVE: Talento; fútbol sala; grupos de edad; efecto de la edad relative

\section{REFERÊNCIAS}

ARAÚJO, T. L. et al. Demanda fisiológica durante um jogo de futebol de salão, através da distância percorrida. Revista da APEF, Londrina, v. II, n. 19, p. 12-20, 1996.

COSTA, V. T. et al. Comparison of relative age of elite athletes participating in the 2008 Brazilian soccer championship series A and B. Motricidade, Portugal, v. 5, n. 3, p. 35-38, 2009. Dísponível em: http://www.revistamotricidade.com/arquivo/2009_vol5_n3/v5n3a05. pdf Acesso em: 20 fev. 2010.

CÔTÉ, J. et al. When "where" is more important than "when": Birthplace and birth date effects on the achievement of sporting expertise. Journal of Sports Sciences, London, v. 24, n. 10, p. 1065-1073, 2006.

DACOSTA, L. (Org.). Atlas do esporte no Brasil. Rio de Janeiro: Shape, 2005.

DELORME, N.; BOICHÉ, J.; RASPAUD, M. Relative age and dropout in french male soccer. Journal of Sports Sciences, London, v. 28, n. 7, p. 717-722, 2010. 
DELORME, N; RASPAUD, M. The relative age effect in young French basketball players: a study on the whole population. Scandinavian Journal of Medicine and Science in Sports, Oxford, n. 19, p. 235-242, 2009.

EDGAR, S.; O'DONOGHUE, P. Season of birth distribution of elite tennis players. Journal of Sports Sciences, London, v. 23, n. 10, p. 1013-1020, 2006.

FOLGADO, H. A. et al. Efeito da idade cronológica na distribuição dos futebolistas por escalões de formação e pelas diferentes posições especificas. Revista Portuguesa de Ciências do Desporto, Porto, v. 6, n. 3, p. 349-355, 2006.

GENEROSI, R. et al. Aspectos morfológicos observados em atletas profissionais do futsal masculino brasileiro. Efdeportes: revista digital, Buenos Aires, ano 13, n. 124, 2008. Disponível em: http://www.efdeportes.com/efd I 24/aspectos-morfologicos-em-atletas-do-futsal-masculino.htm. Acesso em: 18 jan. 2010.

GIL, S. et al. Selection of young soccer players in terms of anthropometric and physiological factors. Journal of Sports Medicine and Physical Fitness, Torino, v. 47, n. I, p. 25-32, 2007.

GLAMSER, F. D.; VICENT, J. The relative age effect among elite american youth soccer players. Journal of Sport Behavior, New York, v. 27, n. I, p. 31-39, 2004.

HELSEN, W. F.; WINCKEL, J. V.; WILLIAMS, M. The relative age effect in youth soccer across Europe. Journal of Sport Sciences, London, v. 23, n. 6, p. 629-636, 2005.

HELSEN, W. F.; STARKES, J. L.; WINCKEL, J. V. Effect of a change in selection year on success in male soccer players. American Journal of Human Biology, New York, v. I2, n. 6, 2000.

JIMÉNEZ, I. P.; PAIN, M. T. G. Relative age effect in spanish association football: its extent and implications for wasted potential. Journal of Sports Sciences, London, v. 26, n. I0, p. 995-1003, 2008.

MACDONALD, D. J. et al. Place but not date of birth influences the development and emergence of athletic talent in American football. Journal of Applied Sport Psychology, London, v. 21 , p. 80-90, 2009.

MARQUES, M. P.; SAMULSKI, D. M. Análise da carreira esportiva de jovens atletas de futebol na transição da fase amadora para a fase profissional: escolaridade, iniciação, contexto sócio-familiar e planejamento da carreira. Revista Brasileira de Educação Física e Esporte, São Paulo, v. 23, n. 2, p. 103-119, 2009.

MORAES, L. C. C. A. et al. Análise do quartil de nascimento de atletas profissionais de futebol. Pensar a Prática, Goiânia, v. 12, n. 3, p. 1-9, 2009. Disponível em: http://www.revistas.ufg. br/index.php/fef/article/view/6683/6186. Acesso em: 20 fev. 2010.

MORALES, J. C. P. et al. Processo de ensino-aprendizagem-treinamento no minibasquetebol. Fitness \& Performance Journal, Rio de Janeiro, n. 8, v. 5, p. 349-359, 2009. 
MUSCH, J.; GRONDIN, S. Unequal competition as an impediment to personal development: a review of the relative age effect in sport. Developmental Review, New York, v. 2 I , n. 2 , p. |47-167, 200|.

MUSCH, J.; HAY, R. The relative age effect in soccer: Cross-cultural evidence for a systematic discrimination against children born late in the competition year. Sociology of Sport Journal, Champaign, v. 16, n. I, p. 54-64, 1999.

QUEIROGA, M. R.; FERREIRA, S. A.; ROMANZINI, M. Perfil antropométrico de atletas de futsal feminino de alto nível competitivo conforme a função tática desempenhada no jogo. Revista Brasileira de Cineantropometria e Desempenho Humano, Florianópolis, v. 7, n. I p. 30-34, 2005. Disponível em: < http://www.rbcdh.ufsc.br/DownloadArtigo.do?artigo=126> acesso em: 01 mar. 2010.

SHERAR, L. B. et al. Do physical maturity and birth date predict talent in male youth ice hockey players? Journal of Sports Sciences, London, v. 25, n. 8, p. 879-886, 2007.

SIMMONS, C.; PAULL, G. C. Season-of-birth bias in association football. Journal of Sports Sciences, London, v. 19, n. 9, p. 677-686, 2001.

TOMPSON, A. H.; BARNSLEY, R. H.; STEBELSKY, G. Born to play ball: the relative age effect and major league baseball. Sociology of Sport Journal, Champaign, n. 8, p. I46- I 5 I, I99।.

VAEYENS, R.; PHILIPPAERTS, R. M.; MALINA, R. M. The relative age effect in soccer: A match-related perspective. Journal of Sports Sciences, London, v. 23, n. 7, p. 747-756, 2005.

VITOR, F. M. et al. Aptidão física de jovens atletas do sexo masculino em relação à idade cronológica e estágio de maturação sexual. Revista Brasileira de Educação Física e Esportes, São Paulo, v. 22, n. 2, p. |39-|48, 2008.

Recebido: 08 jun. 2010

Aprovado: 12 ago. 2011

Endereço para correspondência:

Eduardo Macedo Penna Rua Astolfo Vieira de Resende I49 apto I0 I Bairro Sion Belo Horizonte - MG CEP: 303 I5-5। 0 